\title{
ON THE EXCEPTIONAL SET IN NEVANLINNA'S SECOND FUNDAMENTAL THEOREM IN THE UNIT DISC
}

\author{
Arturo Fernández Arias and Francisco Rodríguez Mateos
}

Abstract

A general example of an analytic function in the unit disc possessing an exceptional set in Nevanlinna's second fundamental theorem is built. It is used to show that some conditions on the size of the exceptional set are sharp, extending analogous results for meromorphic functions in the plane.

\section{Introduction}

We shall use the standard terminology of Nevanlinna theory (see, for example, $[\mathbf{4}],[\mathbf{6}],[\mathbf{7}])$. In $[\mathbf{3}]$ we prove the following theorems, sharpening a result of R. Nevanlinna [7, p. 247] and extending to meromorphic functions in the unit disc analogous results in the plane [1].

Theorem A. Let $F$ be a meromorphic function in the unit disc $\mathcal{D}=$ $\{z|| z \mid<1\}$ and $\lambda \geq 0$ a positive real number. Then the error term $S(r, F)$ in Nevanlinna's second fundamental theorem satisfies

$$
S(r, F)=O\left(\log ^{+} T(r, F)\right)+O\left(\log \frac{1}{1-r}\right)
$$

as $r \rightarrow 1$ outside a set $E_{\lambda}$ such that

$$
\int_{E_{\lambda}} \frac{d r}{(1-r)^{\lambda}}<\infty
$$

Theorem B. Let $F$ be meromorphic in the unit disc and such that

$$
\log \left(\frac{1}{1-r}\right)=o(T(r, F)), \text { as } r \rightarrow 1 .
$$


Then the error term $S(r, F)$ satisfies

$$
S(r, F)=o(T(r, F))
$$

as $r \rightarrow 1$ outside a set $E$, independent of $\lambda$, such that

$$
\int_{E}\left(\frac{1}{1-r}\right)^{\lambda} d r<\infty
$$

for every $\lambda>0$.

Theorem C. Let $F$ be a meromorphic function in the unit disc satisfying (1.3). Then the error term $S(r, F)$ satisfies (1.4) outside a set $E$ which can be contained in a sequence of intervals $\left[r_{n}, r_{n}+\delta_{n}\right]$ such that $r_{n}$ is increasing and tends to 1 and

$$
\delta_{n}<\frac{1-r_{n}}{e \Psi(n)^{2}}, \text { where } \Psi(1)=1, \Psi(n)=e^{\Psi(n-1)} .
$$

In this paper we show that these results are sharp. To do this, we shall give an example of a meromorphic function in the unit disc possessing a suitable exceptional set. The basic ideas of the example go back to W. K. Hayman [5] and A. Fernández [2], although to obtain the desired properties a more complicated construction is needed.

\section{Statement of the results}

Theorem 1. For every function $\phi(r), 0 \leq r<1$, such that

$$
\phi(r)(1-r)^{\lambda} \rightarrow \infty \text {, as } r \rightarrow 1, \text { for all positive } \lambda,
$$

we can construct a meromorphic function F satisfying (1.3) such that

$$
\frac{S(r, F)}{\log \frac{T(r, F)}{1-r}} \rightarrow \infty
$$

and

$$
S(r, F)>2 T(r, F)
$$

in a set $E \subset[0,1]$ satisfying

$$
\int_{E} \phi(r) d r=\infty
$$

This result shows that Theorems A and B are sharp. Next theorem gives a converse result to Theorem $\mathrm{C}$. 
Theorem 2. Let $\Psi(n)$ be defined as in (1.6). Then for every increasing sequence $L(n)$ such that there is no $N$ in $\mathbf{N}$ for which

$$
\Psi(n+N) \geq L(n), \quad n \in \mathbf{N}
$$

there is a meromorphic function in the unit disc $F$, satisfying (1.3), for which we cannot find a sequence of disjoint intervals $\left[r_{n}, r_{n}+\delta_{n}\right]$ such that $r_{n}$ is increasing and tends to one and $\delta_{n}$ satisfies

$$
\delta_{n}<\frac{1-r_{n}}{e L(n)^{2}}
$$

and such that $S(r, F)=o(T(r, F))$ outside the union $\bigcup_{n}\left[r_{n}, r_{n}+\delta_{n}\right]$.

The proofs of Theorems 1 and 2 rely on the following construction, which gives an example of a meromorphic function in the unit disc possessing an exceptional set in Nevanlinna's second fundamental theorem.

Theorem 3. Suppose that $0<\alpha<1$ and that $\left\{r_{n}\right\}$ is a sequence of positive numbers satisfying

$$
r_{n}<1, \quad 1-r_{n+1}<\alpha^{2}\left(1-r_{n}\right) \quad n=1,2, \ldots
$$

Let $\left\{\lambda_{n}\right\}$ be the sequence of integers defined by

$$
\lambda_{n}= \begin{cases}1 & n=1 \\ {\left[\left(\frac{1}{1-r_{n}}\right)^{\lambda_{n-1}^{2}}\right]+1} & n=2,3, \ldots\end{cases}
$$

and $\left\{m_{n}\right\}$ the sequence of integers

$$
m_{n}= \begin{cases}1, & n=1 \\ {\left[\frac{\sqrt{\lambda_{n}}\left(1-r_{n-1}\right)}{1-r_{n}}\right],} & n=2,3, \ldots\end{cases}
$$

Write

$$
F(z)=\sum_{n=1}^{\infty} \sum_{k=0}^{m_{n}-1}\left(\frac{1-r_{n}}{\exp \left(\frac{2 k \pi i}{m_{n}}\right)-z}\right)^{\lambda_{n}}
$$


Then $F(z)$ is analytic in the unit disc $\mathcal{D}$ and satisfies (1.3). Further, if $\left\{s_{n}\right\}$ is the sequence of positive numbers defined by

$$
\frac{1}{1-s_{n}}=\left\{\left(\frac{1}{1-r_{n}}\right)^{\lambda_{n}} 2(n-1) \lambda_{n-1}\right\}^{\frac{1}{\lambda_{n}-\lambda_{n-1}}}
$$

and

$$
s_{n}^{\prime}=s_{n}+\delta_{n}, \quad \delta_{n}=o\left(\frac{1-r_{n}}{\lambda_{n}}\right)
$$

then

$$
\frac{T\left(r, F^{\prime}\right)}{T(r, F)} \rightarrow \infty
$$

and

$$
\frac{T\left(r, F^{\prime}\right)}{\log \frac{T(r, F)}{1-r}} \rightarrow \infty
$$

as $r \rightarrow 1$ in $E=\bigcup_{n}\left[s_{n}, s_{n}^{\prime}\right]$.

\section{Proof of Theorem 3}

We note that, by (2.7), $r_{n} \rightarrow 1$ as $n \rightarrow \infty$.

We consider

$$
z \in \mathcal{D}, \quad z=r e^{i \vartheta}
$$

such that

$$
\alpha\left(1-r_{N}\right)<1-r<1-r_{N}
$$

and note that, from (2.7), this implies

$$
1-r_{N+1}<\alpha(1-r) \text {. }
$$

Next we write

$$
\vartheta_{k, n}=\frac{2 k \pi}{m_{n}}
$$

For each $n>1$ we can find $k_{0}$ such that

$$
\left|e^{i \vartheta_{k_{0}, n}}-z\right| \leq\left|e^{i \vartheta_{k, n}}-z\right|, \quad k=0,1, \ldots, m_{n}-1
$$


Then, if we write

$$
\alpha_{k, n}=\left\{\begin{array}{ll}
\left|\vartheta-\vartheta_{k, n}\right| & \text { if }\left|\vartheta-\vartheta_{k, n}\right| \leq \pi \\
2 \pi-\left|\vartheta-\vartheta_{k, n}\right| & \text { if }\left|\vartheta-\vartheta_{k, n}\right| \geq \pi
\end{array} \quad k=0,1, \ldots, m_{n}-1\right.
$$

we have

$$
\begin{array}{rlrl}
\alpha_{k_{0}, n} & \leq \frac{\pi}{m_{n}} \\
\alpha_{k, n} & \geq \frac{\left(2\left|k-k_{0}\right|-1\right) \pi}{m_{n}}, & & \text { if } 0<\left|k-k_{0}\right| \leq \frac{m_{n}-1}{2} \\
\alpha_{k, n} & \geq \frac{\left(2\left(m_{n}-\left|k-k_{0}\right|\right)-1\right) \pi}{m_{n}}, & \text { if }\left|k-k_{0}\right| \geq \frac{m_{n}+1}{2}
\end{array}
$$

and, in any case, $\alpha_{k, n} \leq \pi$.

Now we consider $n$ given large and, for the sake of simplicity and without loss of generality, suppose that $k_{0}=0$.

Then we have, for $1 \leq k \leq\left(m_{n}-1\right) / 2$,

$$
\alpha_{k, n} \geq \frac{(2 k-1) \pi}{m_{n}} \geq \frac{\sqrt{k} \pi}{m_{n}} \sim \frac{\sqrt{k} \pi\left(1-r_{n}\right)}{\sqrt{\lambda_{n}}\left(1-r_{n-1}\right)},
$$

so that

$$
\begin{aligned}
\left|e^{i \vartheta_{k, n}}-r e^{i \vartheta}\right|^{2} & =1+r^{2}-2 r \cos \left(\vartheta_{k, n}-\vartheta\right) \\
& =1+r^{2}-2 r \cos \alpha_{k, n}>1+r^{2}-2 r\left(1-\frac{\alpha_{k, n}^{2}}{2}+\frac{\alpha_{k, n}^{4}}{4 !}\right) \\
& =(1-r)^{2}+r \alpha_{k, n}^{2}\left(1-\frac{\alpha_{k, n}^{2}}{12}\right) \\
& \geq(1-r)^{2}+\frac{r k \pi^{2}\left(1-r_{n}\right)^{2}}{\lambda_{n}\left(1-r_{n-1}\right)^{2}}\left(1-\frac{\pi^{2}}{12}\right) \\
& =(1-r)^{2}+\frac{c r k\left(1-r_{n}\right)^{2}}{\lambda_{n}\left(1-r_{n-1}\right)^{2}}, \quad c=\pi^{2}\left(1-\frac{\pi^{2}}{12}\right) .
\end{aligned}
$$

We note that for $\left(m_{n}+1\right) / 2 \leq k \leq m_{n}-1$ a similar result holds in terms of $k^{\prime}=m_{n}-k$, and that for $k_{o} \neq 0$ the only change needed in (3.3) is writing $\left|k-k_{0}\right|$ instead of $k$.

We have

$$
\left|\frac{1-r_{n}}{e^{i \vartheta_{k, n}}-z}\right|^{\lambda_{n}}=\left(\frac{1-r_{n}}{1-r}\right)^{\lambda_{n}}\left(\frac{(1-r)^{2}}{\left|e^{i \vartheta_{k, n}}-z\right|^{2}}\right)^{\frac{\lambda_{n}}{2}}
$$


and from (3.3)

$$
\begin{aligned}
\left(\frac{(1-r)^{2}}{\left|e^{i \vartheta_{k, n}}-z\right|^{2}}\right)^{\frac{\lambda_{n}}{2}} & <\left(\frac{(1-r)^{2}}{(1-r)^{2}+\frac{c r k\left(1-r_{n}\right)^{2}}{\lambda_{n}\left(1-r_{n-1}\right)^{2}}}\right)^{\frac{\lambda_{n}}{2}} \\
& =\left(1+\frac{c r k\left(1-r_{n}\right)^{2}}{\lambda_{n}\left(1-r_{n-1}\right)^{2}(1-r)^{2}}\right)^{-\frac{\lambda_{n}}{2}}
\end{aligned}
$$

Next we show that in the sum

$$
\sum_{k=0}^{m_{n}-1}\left|\frac{1-r_{n}}{e^{i \vartheta_{k, n}}-z}\right|^{\lambda_{n}}=\left|\frac{1-r_{n}}{e^{i \vartheta_{k_{0}, n}}-z}\right|^{\lambda_{n}}+\sum_{\substack{k=0 \\ k \neq k_{0}}}^{m_{n}-1}\left|\frac{1-r_{n}}{e^{i \vartheta_{k, n}}-z}\right|^{\lambda_{n}}
$$

for $n$ large the main term is that one corresponding to $k_{0}$.

We recall that, from Euler's summation formula, we have for any real function $f$ possessing continuous derivative $f^{\prime}$ in the interval $[y, x], 0<$ $y<x$,

$$
\begin{aligned}
\sum_{\substack{y<k \leq x \\
k \in \mathbf{N}}} f(k)= & \int_{y}^{x} f(t) d t+\int_{y}^{x}(t-[t]) f^{\prime}(t) d t \\
& +f(x)([x]-x)-f(y)([y]-y),
\end{aligned}
$$

so that taking in this formula

$$
\begin{aligned}
f(t) & =\left(1+\frac{\operatorname{crt}\left(1-r_{n}\right)^{2}}{\lambda_{n}\left(1-r_{n-1}\right)^{2}(1-r)^{2}}\right)^{-\frac{\lambda_{n}}{2}} \\
y & =1, \quad x=\left[\frac{m_{n}-1}{2}\right]
\end{aligned}
$$

and noting that for this election

$$
\begin{aligned}
& {[x]-x=[y]-y=0} \\
& f^{\prime}(t)<0 \text { for } t>0
\end{aligned}
$$


The EXCeptional SET In Nevanlinna theory In the DisC 141

we have

$\sum_{k=2}^{x} f(k) \leq \frac{2 \lambda_{n}(1-r)^{2}\left(1-r_{n-1}\right)^{2}}{\left(\lambda_{n}-2\right) c r\left(1-r_{n}\right)^{2}}\left(1+\frac{c r\left(1-r_{n}\right)^{2}}{\lambda_{n}\left(1-r_{n-1}\right)^{2}(1-r)^{2}}\right)^{-\frac{\lambda_{n}}{2}+1}$,

so that

$$
\begin{aligned}
\sum_{k=1}^{x} f(k) & <\left(1+\frac{c r\left(1-r_{n}\right)^{2}}{\lambda_{n}\left(1-r_{n-1}\right)^{2}(1-r)^{2}}\right)^{-\frac{\lambda_{n}}{2}} \\
& \left\{1+\frac{2 \lambda_{n}(1-r)^{2}\left(1-r_{n-1}\right)^{2}}{\left(\lambda_{n}-2\right) c r\left(1-r_{n}\right)^{2}}\left(1+\frac{c r\left(1-r_{n}\right)^{2}}{\lambda_{n}\left(1-r_{n-1}\right)^{2}(1-r)^{2}}\right)\right\} .
\end{aligned}
$$

From (3.1) we see that $N \rightarrow \infty$ is equivalent to $r \rightarrow 1$ and then, since (2.7) and (2.8) imply that $\lambda_{n}\left(1-r_{n-1}\right)^{2} \rightarrow \infty$ as $n \rightarrow \infty$, we have

$$
\frac{c r\left(1-r_{N}\right)^{2}}{\lambda_{N}\left(1-r_{N-1}\right)^{2}(1-r)^{2}} \rightarrow 0, \text { as } r \rightarrow 1
$$

We also have

$$
\frac{2 \lambda_{N}(1-r)^{2}\left(1-r_{N-1}\right)^{2}}{\left(\lambda_{N}-2\right) c r\left(1-r_{N}\right)^{2}} \rightarrow 0
$$

and

$$
\left(1+\frac{c r\left(1-r_{N}\right)^{2}}{\lambda_{N}\left(1-r_{N-1}\right)^{2}(1-r)^{2}}\right)^{-\frac{\lambda_{N}}{2}} \rightarrow 0,
$$

so that we obtain, recalling that we are supposing that $k_{0}=0$,

$$
\begin{gathered}
\sum_{k=1}^{m_{N}-1}\left|\frac{1-r_{N}}{e^{i \vartheta_{k, N}}-z}\right|^{\lambda_{N}} \sim 2 \sum_{k=1}^{\left[\frac{m_{N}-1}{2}\right]}\left(\frac{1-r_{N}}{1-r}\right)^{\lambda_{N}}\left|\frac{(1-r)^{2}}{\left|e^{i \vartheta_{k, N}}-z\right|^{2}}\right|^{\frac{\lambda_{N}}{2}} \\
<2\left(\frac{1-r_{N}}{1-r}\right)^{\lambda_{N}} \sum_{k=1}^{x} f(k)=o\left\{\left(\frac{1-r_{N}}{1-r}\right)^{\lambda_{N}}\right\}
\end{gathered}
$$

We also have

$$
\left|\frac{1-r_{N}}{1-z}\right|^{\lambda_{N}}<\left(\frac{1-r_{N}}{1-r}\right)^{\lambda_{N}}
$$


so that

$$
\sum_{k=0}^{m_{N}-1}\left|\frac{1-r_{N}}{e^{i \vartheta_{k, N}}-z}\right|^{\lambda_{N}}<\left(\frac{1-r_{N}}{1-r}\right)^{\lambda_{N}}(1+o(1)) .
$$

Next we consider $r$ sufficiently close to 1 so that

$$
\sum_{k=0}^{m_{N}-1}\left|\frac{1-r_{N}}{e^{i \vartheta_{k, N}}-z}\right|^{\lambda_{N}}<2\left(\frac{1-r_{N}}{1-r}\right)^{\lambda_{N}}
$$

We write

$$
F(z)=\sum_{1}+\sum_{2}+\sum_{3}
$$

where

$$
\begin{aligned}
\left|\sum_{1}\right| & =\left|\sum_{n=1}^{N-1} \sum_{k=0}^{m_{n}-1}\left(\frac{1-r_{n}}{e^{i \vartheta_{k, n}}-z}\right)^{\lambda_{n}}\right| \\
& \leq \sum_{n=1}^{N-1} \sum_{k=0}^{m_{n}-1}\left(\frac{1-r_{n}}{1-r}\right)^{\lambda_{n}} \\
& =\sum_{n=1}^{N-1} m_{n}\left(\frac{1-r_{n}}{1-r}\right)^{\lambda_{n}}<\sum_{n=1}^{N-1} m_{n}\left(\frac{1}{1-r}\right)^{\lambda_{n}} \\
& <(N-1) m_{N-1}\left(\frac{1}{1-r}\right)^{\lambda_{N-1}} \\
& \sim(N-1) \frac{\sqrt{\lambda_{N-1}}\left(1-r_{N-2}\right)}{\left(1-r_{N-1}\right)}\left(\frac{1}{1-r}\right)^{\lambda_{N-1}} \\
& <(N-1) \sqrt{\lambda_{N-1}}\left(\frac{1}{1-r}\right)^{\lambda_{N-1}+1},
\end{aligned}
$$

since, for $n$ large, $m_{n}$ is increasing by (2.8) and (2.9), and by (2.7) and (3.1) we have $\left(1-r_{N-1}\right)>(1-r)$.

$$
\begin{aligned}
\left|\sum_{2}\right| & =\left|\sum_{k=0}^{m_{N}-1}\left(\frac{1-r_{N}}{e^{i \vartheta_{k, N}}-z}\right)^{\lambda_{N}}\right| \\
& \leq\left(\frac{1-r_{N}}{1-r}\right)^{\lambda_{N}}(1+o(1)) \leq 2\left(\frac{1-r_{N}}{1-r}\right)^{\lambda_{N}} .
\end{aligned}
$$


The EXCeptional Set in Nevanlinna theORY IN the DisC 143

$$
\begin{aligned}
\left|\sum_{3}\right| & =\left|\sum_{n=N+1}^{\infty} \sum_{k=0}^{m_{n}-1}\left(\frac{1-r_{n}}{e^{i \vartheta_{k, n}}-z}\right)^{\lambda_{n}}\right| \\
& \leq \sum_{n=N+1}^{\infty} m_{n}\left(\frac{1-r_{n}}{1-r}\right)^{\lambda_{n}} \\
& \sim \sum_{n=N+1}^{\infty} \frac{\sqrt{\lambda_{n}}\left(1-r_{n-1}\right)}{\left(1-r_{n}\right)}\left(\frac{1-r_{n}}{1-r}\right)^{\lambda_{n}} \\
& <\frac{1}{1-r} \sum_{n=N+1}^{\infty} \sqrt{\lambda_{n}}\left(\frac{1-r_{n}}{1-r}\right)^{\lambda_{n}-1} \\
& <\frac{1}{1-r} \sum_{n=N+1}^{\infty}\left(\lambda_{n}-1\right) \alpha^{\lambda_{n}-1} \\
& <\frac{1}{1-r} \sum_{n=1}^{\infty} n \alpha^{n}=\frac{c_{1}}{1-r}, \quad c_{1}=\frac{\alpha}{(1-\alpha)^{2}},
\end{aligned}
$$

since from (2.7) and (3.1) we have

$$
\frac{1-r_{n}}{1-r}<\alpha, \quad n=N+1, N+2, \ldots
$$

From (3.6), (3.7) and (3.8) we conclude that $F(z)$ converges in any compact subset of $\mathcal{D}$, so that it is analytic in $\mathcal{D}$.

Writing

$$
f(z)=F^{\prime}(z)=\sum_{1}{ }^{\prime}+\sum_{2}{ }^{\prime}+\sum_{3}{ }^{\prime}
$$

we have, as in (3.6) and (3.8),

$$
\begin{aligned}
\left|\sum_{1}\right| & =\left|\sum_{n=1}^{N-1} \lambda_{n} \sum_{k=0}^{m_{n}-1}\left(\frac{1-r_{n}}{e^{i \vartheta_{k, n}}-z}\right)^{\lambda_{n}} \frac{1}{e^{i \vartheta_{k, n}}-z}\right| \\
& \leq \sum_{n=1}^{N-1} \lambda_{n} m_{n}\left(\frac{1-r_{n}}{1-r}\right)^{\lambda_{n}} \frac{1}{1-r} \\
& <(N-1) \lambda_{N-1} m_{N-1}\left(\frac{1}{1-r}\right)^{\lambda_{N-1}} \frac{1}{1-r} \\
& <(N-1) \lambda_{N-1}^{3 / 2}\left(\frac{1}{1-r}\right)^{\lambda_{N-1}+2}(1+o(1)) .
\end{aligned}
$$




$$
\begin{aligned}
\left|\sum_{3}\right| & =\left|\sum_{n=N+1}^{\infty} \lambda_{n} \sum_{k=0}^{m_{n}-1}\left(\frac{1-r_{n}}{e^{i \vartheta_{k, n}}-z}\right)^{\lambda_{n}} \frac{1}{\left(e^{i \vartheta_{k, n}}-z\right)}\right| \\
& \leq \sum_{n=N+1}^{\infty} \lambda_{n} m_{n}\left(\frac{1-r_{n}}{1-r}\right)^{\lambda_{n}} \frac{1}{1-r} \\
& \sim \sum_{n=N+1}^{\infty} \lambda_{n} \frac{\sqrt{\lambda_{n}}\left(1-r_{n-1}\right)}{\left(1-r_{n}\right)}\left(\frac{1-r_{n}}{1-r}\right)^{\lambda_{n}} \frac{1}{1-r} \\
& <\frac{1}{(1-r)^{2}} \sum_{n=N+1}^{\infty} \lambda_{n}\left(\lambda_{n}-1\right) \alpha^{\lambda_{n}-1} \\
& <\frac{1}{(1-r)^{2}} \sum_{n=1}^{\infty} n(n-1) \alpha^{n-1} \\
& =\frac{c_{2}}{(1-r)^{2}}, \quad c_{2}=\frac{\alpha}{(1-\alpha)^{3}} .
\end{aligned}
$$

For $s_{n}$ as defined in (2.11) we have

$$
\left(\frac{1-r_{n}}{1-s_{n}}\right)^{\lambda_{n}}=2(n-1) \lambda_{n-1}\left(\frac{1}{1-s_{n}}\right)^{\lambda_{n-1}}
$$

and $s_{n}>r_{n}$. Next we show that

$$
\frac{1}{1-s_{n}} \sim \frac{1}{1-r_{n}}, \text { as } n \rightarrow \infty
$$

i.e.

$$
\lim _{n \rightarrow \infty} \frac{\left(\frac{1}{1-r_{n}}\right)^{\frac{\lambda_{n}}{\lambda_{n}-\lambda_{n-1}}}(2(n-1))^{\frac{1}{\lambda_{n}-\lambda_{n}-1}} \lambda_{n-1}{ }^{\frac{1}{\lambda_{n}-\lambda_{n-1}}}}{\frac{1}{1-r_{n}}}=1 .
$$

The logarithm of the left hand side is

$$
\left(\frac{\lambda_{n}}{\lambda_{n}-\lambda_{n-1}}-1\right) \log \left(\frac{1}{1-r_{n}}\right)+\frac{\log (2(n-1))}{\lambda_{n}-\lambda_{n-1}}+\frac{\log \lambda_{n-1}}{\lambda_{n}-\lambda_{n-1}} .
$$

Since $\frac{\lambda_{n-1}}{\lambda_{n}}$ and $\frac{n}{\lambda_{n}}$ tend to zero as $n$ tends to infinity by (2.8), the last two terms tend to zero. The first term satisfies

$$
\begin{aligned}
\left(\frac{\lambda_{n}}{\lambda_{n}-\lambda_{n-1}}-1\right) \log \left(\frac{1}{1-r_{n}}\right) & =\frac{\lambda_{n-1} \log \left(\frac{1}{1-r_{n}}\right)}{\lambda_{n}-\lambda_{n-1}} \\
& <\frac{\log \lambda_{n}}{\lambda_{n}-\lambda_{n-1}} \rightarrow 0,
\end{aligned}
$$


as $n \rightarrow \infty$, by (2.8).

Next we show that if $r \in\left[s_{N}, s_{N}^{\prime}\right]$ for $N$ large then $r$ satisfies (3.1).

We have

$$
\begin{gathered}
r_{N} \leq s_{N} \leq r<s_{N}+\delta_{N} \\
1-s_{N}-\delta_{N}<1-r \leq 1-r_{N} \\
\frac{1-s_{N}-\frac{1-r_{N}}{\lambda_{N}}}{1-r_{N}} \leq \frac{1-r}{1-r_{N}} \leq 1
\end{gathered}
$$

but

$$
1-s_{N} \sim 1-r_{N}
$$

and

$$
\frac{1-r_{N}}{\lambda_{N}} \rightarrow 0,
$$

so that we can find $\varepsilon>0$ such that $1-\varepsilon>\alpha$ and $N_{0}$ such that for $N \geq N_{0}$

$$
1-r_{N}>1-r \geq(1-\varepsilon)\left(1-r_{N}\right)>\alpha\left(1-r_{N}\right) .
$$

Next we prove that as $r \rightarrow 1$ through the intervals $\left[s_{n}, s_{n}^{\prime}\right]$ we have

$$
\left(\frac{1-r_{n}}{1-r}\right)^{\lambda_{n}} \sim\left(\frac{1-r_{n}}{1-s_{n}}\right)^{\lambda_{n}}
$$

or equivalently

$$
\left(\frac{1-s_{n}^{\prime}}{1-s_{n}}\right)^{\lambda_{n}} \rightarrow 1
$$

In fact

$$
\left(\frac{1-s_{n}-\delta_{n}}{1-s_{n}}\right)^{\lambda_{n}}=\left\{\left(1-\frac{1}{\frac{1-s_{n}}{\delta_{n}}}\right)^{\frac{1-s_{n}}{\delta_{n}}}\right\}^{\delta_{n} \frac{\lambda_{n}}{1-s_{n}}} \rightarrow e^{0}=1
$$

by (2.12) and (3.12). We also have for $r \in\left[s_{n}, s_{n}^{\prime}\right]$

$$
\left(\frac{1-r_{n}}{1-r}\right)^{\lambda_{n-1}} \sim\left(\frac{1-r_{n}}{1-s_{n}}\right)^{\lambda_{n-1}} .
$$

From (3.11) and (3.13) we deduce

$$
\left(\frac{1-r_{n}}{1-r}\right)^{\lambda_{n}} \sim 2(n-1) \lambda_{n-1}\left(\frac{1}{1-r}\right)^{\lambda_{n-1}} .
$$


We have seen that if $z=r e^{i \vartheta}$ and $r \in\left[s_{N}, s_{N}^{\prime}\right]$ for $N$ large $r$ satisfies (3.1) and we can apply (3.6), (3.7) and (3.8) to obtain

$$
|F(z)|<4(N-1) \lambda_{N-1}\left(\frac{1}{1-r}\right)^{\lambda_{N-1}+1}(1+o(1)) .
$$

Hence

$$
T(r, F)<\lambda_{N-1} \log \left(\frac{1}{1-r}\right)(1+o(1))
$$

Next we consider $z$ satisfying

$$
\alpha_{k_{0}, N} \leq \frac{\pi\left(1-r_{N-1}\right)}{m_{N}}
$$

Then

$$
\begin{aligned}
\left|e^{i \vartheta_{k_{0}, N}}-z\right|^{2} & =1+r^{2}-2 r \cos \alpha_{k_{0}, N} \\
& <1+r^{2}-2 r\left(1-\frac{\alpha_{k_{0}, N}^{2}}{2}\right)=(1-r)^{2}+r \alpha_{k_{0}, N}^{2} \\
& <(1-r)^{2}+\frac{r \pi^{2}\left(1-r_{N-1}\right)^{2}}{m_{N}^{2}} \\
& \sim(1-r)^{2}+\frac{r \pi^{2}\left(1-r_{N-1}\right)^{2}\left(1-r_{N}\right)^{2}}{\lambda_{N}\left(1-r_{N-1}\right)^{2}} \\
& =(1-r)^{2}\left(1+\frac{r \pi^{2}}{\lambda_{N}}\left(\frac{1-r_{N}}{1-r}\right)^{2}\right) \\
& <(1-r)^{2}\left(1+\frac{r \pi^{2}}{\lambda_{N} \alpha^{2}}\right)
\end{aligned}
$$

since, by $(3.1),(1-r)>\alpha\left(1-r_{N}\right)$. Thus, for $N$ large we obtain (3.17)

$$
\begin{aligned}
\left(\frac{(1-r)^{2}}{\left|e^{i \vartheta_{k_{0}}, N}-z\right|^{2}}\right)^{\frac{\lambda_{N}}{2}} & >\left(\frac{(1-r)^{2}}{(1-r)^{2}\left(1+\frac{r \pi^{2}}{\lambda_{N} \alpha^{2}}\right)}\right)^{\frac{\lambda_{N}}{2}} \\
& =\left(1+\frac{r \pi^{2}}{\lambda_{N} \alpha^{2}}\right)^{-\frac{\lambda_{N}}{2}}>\exp \left(-\frac{\pi^{2}}{2 \alpha^{2}}\right)=K,
\end{aligned}
$$


so that

$$
\begin{aligned}
\lambda_{N} & \left|\frac{1-r_{N}}{e^{i \vartheta_{k_{0}, N}}-z}\right|^{\lambda_{N}} \frac{1}{\left|e^{i \vartheta_{k_{0}, N}}-z\right|} \\
& =\lambda_{N}\left(\frac{1-r_{N}}{1-r}\right)^{\lambda_{N}}\left(\frac{(1-r)^{2}}{\left|e^{i \vartheta_{k_{0}, N}}-z\right|^{2}}\right)^{\frac{\lambda_{N}}{2}} \frac{1}{\left|e^{i \vartheta_{k_{0}, N}}-z\right|} \\
& >\lambda_{N}\left(\frac{1-r_{N}}{1-r}\right)^{\lambda_{N}}\left(1+\frac{r \pi^{2}}{\lambda_{N} \alpha^{2}}\right)^{-\frac{\lambda_{N}}{2}} \frac{1}{(1-r) \sqrt{1+\frac{r \pi^{2}}{\lambda_{N} \alpha^{2}}}},
\end{aligned}
$$

and from (3.17) and the fact that

$$
\frac{r \pi^{2}}{\lambda_{N} \alpha^{2}} \rightarrow 0
$$

we can find for $N$ large a constant $K_{1}>0$, independent of $N$, such that

$$
\lambda_{N}\left|\frac{1-r_{N}}{e^{i \vartheta_{k_{0}, N}}-z}\right|^{\lambda_{N}} \frac{1}{\left|e^{i \vartheta_{k_{0}, N}}-z\right|}>K_{1} \frac{\lambda_{N}}{1-r}\left(\frac{1-r_{N}}{1-r}\right)^{\lambda_{N}} .
$$

From (3.4) we have

$$
\begin{aligned}
& \left|\lambda_{N} \sum_{k \neq k_{0}}\left(\frac{1-r_{N}}{e^{i \vartheta_{k, N}}-z}\right)^{\lambda_{N}} \frac{1}{\left(e^{i \vartheta_{k, N}}-z\right)}\right| \\
& \quad \leq \frac{\lambda_{N}}{1-r} \sum_{k \neq k_{0}}\left|\frac{1-r_{N}}{e^{i \vartheta_{k, N}}-z}\right|^{\lambda_{N}}=\frac{\lambda_{N}}{1-r} o\left(\frac{1-r_{N}}{1-r}\right)^{\lambda_{N}},
\end{aligned}
$$

so that from (3.18) and (3.19) we obtain

$$
\begin{aligned}
\left|\sum_{2} \prime\right| & \left|\lambda_{N} \sum_{k=0}^{m_{N}-1}\left(\frac{1-r_{N}}{e^{i \vartheta_{k, N}}-z}\right)^{\lambda_{N}} \frac{1}{\left(e^{i \vartheta_{k, N}}-z\right)}\right| \\
\geq & \lambda_{N}\left|\frac{1-r_{N}}{e^{i \vartheta_{k_{0}, N}}-z}\right|^{\lambda_{N}} \frac{1}{\left|e^{i \vartheta_{k_{0}, N}}-z\right|} \\
& -\left|\sum_{k \neq k_{0}} \lambda_{N}\left(\frac{1-r_{N}}{e^{i \vartheta_{k, N}}-z}\right)^{\lambda_{N}} \frac{1}{\left(e^{i \vartheta_{k, N}}-z\right)}\right| \\
\geq & K_{1} \frac{\lambda_{N}}{1-r}\left(\frac{1-r_{N}}{1-r}\right)^{\lambda_{N}}-o\left\{\frac{\lambda_{N}}{1-r}\left(\frac{1-r_{N}}{1-r}\right)^{\lambda_{N}}\right\} \\
\sim & K_{1} \frac{\lambda_{N}}{1-r}\left(\frac{1-r_{N}}{1-r}\right)^{\lambda_{N}} .
\end{aligned}
$$


For $r \in\left[s_{N}, s_{N}^{\prime}\right]$ and $z=r e^{i \vartheta}$ satisfying (3.16) we have for $N$ large, from (3.9), (3.10) and (3.20),

$$
|f(z)| \geq K_{1} \frac{\lambda_{N}}{1-r}\left(\frac{1-r_{N}}{1-r}\right)^{\lambda_{N}}(1+o(1)) .
$$

For every $z=r e^{i \vartheta}$ we can find $k_{0} \in\left\{0,1, \ldots, m_{N}-1\right\}$ such that

$$
\alpha_{k_{0}, N} \leq \frac{\pi}{m_{N}}
$$

and, for $|z|=r, k_{0}$ is the same for every $z=r e^{i \vartheta}$ in an arc of amplitude $2 \pi / m_{N}$ around $r e^{i \vartheta_{k_{0}, N}}$. For (3.16) to hold it is sufficient that $z$ be in an arc of amplitude $\frac{2 \pi\left(1-r_{N-1}\right)}{m_{N}}$ centered at $r e^{i \vartheta_{k_{0}, N}}$. Therefore

$$
\begin{aligned}
& T(r, f)=\frac{1}{2 \pi} \int_{0}^{2 \pi} \log ^{+}\left|f\left(r e^{i \vartheta}\right)\right| d \vartheta \\
& =\frac{1}{2 \pi} \sum_{k=0}^{m_{N}-1} \int_{\vartheta_{k, N}}^{\vartheta_{k+1, N}} \log ^{+}\left|f\left(r e^{i \vartheta}\right)\right| d \vartheta \\
& \geq \frac{1}{2 \pi} \sum_{k=0}^{m_{N}-1}\left\{\int_{\vartheta_{k, N}}^{\vartheta_{k, N}+\frac{\pi\left(1-r_{N-1}\right)}{m_{N}}} \log \left|f\left(r e^{i \vartheta}\right)\right| d \vartheta\right. \\
& \left.+\int_{\vartheta_{k+1, N}-\frac{\pi\left(1-r_{N-1}\right)}{m_{N}}}^{\vartheta_{k+1, N}} \log \left|f\left(r e^{i \vartheta}\right)\right| d \vartheta\right\} \\
& \geq \frac{1}{2 \pi} \sum_{k=0}^{m_{N}-1} \frac{2 \pi\left(1-r_{N-1}\right)}{m_{N}}\left\{\log \lambda_{N}+\log \left(\frac{1-r_{N}}{1-r}\right)^{\lambda_{N}}\right. \\
& \left.+\log \left(\frac{1}{1-r}\right)+\log K_{1}\right\} \\
& \sim\left(1-r_{N-1}\right)\left\{\lambda_{N-1}^{2} \log \left(\frac{1}{1-r}\right)+\lambda_{N-1} \log \left(\frac{1}{1-r}\right)\right. \\
& \left.+\log \left(2 N \lambda_{N-1}\right)+\log \left(\frac{1}{1-r}\right)\right\} \\
& \sim\left(1-r_{N-1}\right) \lambda_{N-1}^{2} \log \left(\frac{1}{1-r}\right) \text {, }
\end{aligned}
$$

since from $r \in\left[s_{N}, s_{N}^{\prime}\right]$ and (3.12) we deduce

$$
\left(\frac{1}{1-r_{N}}\right) \sim\left(\frac{1}{1-r}\right) \text {. }
$$


Hence, from (3.15) and (3.21) we obtain

$$
\frac{T(r, f)}{T(r, F)}>\frac{\left(1-r_{N-1}\right) \lambda_{N-1}^{2} \log \left(\frac{1}{1-r}\right)}{\lambda_{N-1} \log \left(\frac{1}{1-r}\right)}=\left(1-r_{N-1}\right) \lambda_{N-1} \rightarrow \infty
$$

by (2.8), as $r$ tends to one in $E=\bigcup_{N}\left[s_{N}, s_{N}^{\prime}\right]$.

Since from (3.21) we have

$$
\log \left(\frac{1}{1-r}\right)=o(T(r, f))
$$

as $r \rightarrow 1$ in $E$, we also have

$$
\frac{T(r, f)}{\log \frac{T(r, F)}{1-r}} \rightarrow \infty
$$

as $r \rightarrow 1$ in $E$.

Finally we show that $F(z)$ satisfies (1.3). In fact, from (3.6) and (3.8), and with the same arguments used to obtain (3.20), we deduce for $z=r e^{i \vartheta}$ satisfying (3.1) and (3.16) and $N$ large

$$
|F(z)| \geq K_{2}\left(\frac{1-r_{N}}{1-r}\right)^{\lambda_{N}}-(N-1) \sqrt{\lambda_{N-1}}\left(\frac{1}{1-r}\right)^{\lambda_{N-1}+1}-\frac{C_{1}}{1-r}
$$

for a certain constant $K_{2}>0$ independent of $N$.

Then, for $r \in\left[s_{N}, s_{N}^{\prime}\right]$ we obtain, from (3.14) and following similar computations to those in (3.21)

$$
T(r, F) \geq\left(1-r_{N-1}\right) \lambda_{N-1} \log \left(\frac{1}{1-r}\right) .
$$

For $s_{N}^{\prime}<r<r_{N+1}$ we have

$$
\begin{aligned}
\left(\frac{1-r_{N}}{1-r}\right)^{\lambda_{N}} & =\left(\frac{1-r_{N}}{1-s_{N}^{\prime}}\right)^{\lambda_{N}}\left(\frac{1-s_{N}^{\prime}}{1-r}\right)^{\lambda_{N}} \\
& \sim 2(N-1) \lambda_{N-1}\left(\frac{1}{1-s_{N}^{\prime}}\right)^{\lambda_{N-1}}\left(\frac{1-s_{N}^{\prime}}{1-r}\right)^{\lambda_{N}} \\
& >2(N-1) \lambda_{N-1}\left(\frac{1}{1-r}\right)^{\lambda_{N-1}}
\end{aligned}
$$


and we also obtain (3.22).

Finally, for $r_{N}<r<s_{N}$ we have

$$
\begin{aligned}
T(r, F) & \geq T\left(r_{N}, F\right) \geq\left(1-r_{N-2}\right) \lambda_{N-2} \log \frac{1}{1-r_{N}} \\
& \geq\left(1-r_{N-2}\right) \lambda_{N-2} \log \frac{1}{1-r}-\left(1-r_{N-2}\right) \lambda_{N-2} \log \frac{1-r_{N}}{1-s_{N}},
\end{aligned}
$$

but

$$
\begin{aligned}
(1- & \left.r_{N-2}\right) \lambda_{N-2} \log \frac{1-r_{N}}{1-s_{N}} \\
& =\frac{\left(1-r_{N-2}\right) \lambda_{N-2}}{\lambda_{N}-\lambda_{N-1}}\left\{\lambda_{N-1} \log \frac{1}{1-r_{N}}+\log \left(2(N-1) \lambda_{N-1}\right)\right\}
\end{aligned}
$$

so that this term tends to zero as $N$ tends to infinity.

Thus we conclude that for any $r$ close enough to 1 we have

$$
T(r, F) \geq\left\{\left(1-r_{N-2}\right) \lambda_{N-2} \log \frac{1}{1-r}\right\}(1+o(1)),
$$

and therefore

$$
\lim _{r \rightarrow 1} \frac{T(r, F)}{\log \frac{1}{1-r}} \geq \lim _{r \rightarrow 1}\left(1-r_{N-2}\right) \lambda_{N-2}=\infty .
$$

This completes the proof of Theorem 3 .

\section{Proof of Theorem 1}

Since to prove Theorem 1 we shall make use of Theorem 3, we first show that the set $E$ is in fact exceptional for the function $F(z)$.

We make use of the following two inequalities

$$
\begin{gathered}
S(r, F) \geq m\left(r, \frac{F^{\prime}}{F}\right) \\
m\left(r, F^{\prime}\right) \leq m\left(r, \frac{F^{\prime}}{F}\right)+m(r, F),
\end{gathered}
$$

so that by (2.13) we deduce

$$
\begin{aligned}
S(r, F) & \geq m\left(r, F^{\prime}\right)-m(r, F) \\
& =T\left(r, F^{\prime}\right)-T(r, F)=T\left(r, F^{\prime}\right)(1+o(1))
\end{aligned}
$$


for $r \in\left[s_{n}, s_{n}+\delta_{n}\right]$ and $n$ large, and we deduce from (2.14) and (4.3)

$$
\frac{S(r, F)}{\log \frac{T(r, F)}{1-r}} \geq \frac{T\left(r, F^{\prime}\right)(1+o(1))}{\log \frac{T(r, F)}{1-r}} \rightarrow \infty
$$

as $n \rightarrow \infty$, which is $(2.2)$.

From (2.13) we also have, for $r \in\left[s_{n}, s_{n}+\delta_{n}\right]$ and $n$ large,

$$
m\left(r, F^{\prime}\right)=T\left(r, F^{\prime}\right) \geq 3 T(r, F)=3 m(r, F)
$$

and, from (4.1) and (4.2), we conclude

$$
S(r, F) \geq m\left(r, \frac{F^{\prime}}{F}\right) \geq 2 m(r, F)=2 T(r, F)
$$

which is (2.3).

We can assume without loss of generality that $\phi(r)$ is increasing for $r$ close to 1 . In fact, by (2.1), for every positive integer $N$ there exists $t_{N}$ such that

$$
\phi(r) \geq\left(\frac{1}{1-r}\right)^{N}, \quad r \geq t_{N}
$$

where we can clearly assume that $1-t_{N+1} \leq \alpha\left(1-t_{N}\right)$, for a certain $0<\alpha<1$. Then, if we define

$$
\phi_{1}(r)=\left(\frac{1}{1-r}\right)^{N}, \quad t_{N} \leq r<t_{N+1}
$$

for $r \geq t_{1}, \phi_{1}(r)$ is increasing and satisfies (2.1) and also $\phi_{1}(r) \leq \phi(r)$. Hence it is enough to prove (2.4) with $\phi_{1}(r)$ instead of $\phi(r)$.

We have shown that the function $F(z)$ defined in (2.10) satisfies (2.2) and (2.3) as $r$ tends to 1 through the sequence of intervals $\left[s_{n}, s_{n}+\delta_{n}\right]$ defined by (2.11) and (2.12). Thus it is enough to prove (2.4) for the set $E=\bigcup_{n}\left[s_{n}, s_{n}+\delta_{n}\right]$ and for this it is enough to show that

$$
\sum_{n} \phi\left(s_{n}\right) \delta_{n}=\infty
$$

as this implies (2.4) since $\phi$ is increasing.

Next we show that we can choose $\left\{r_{n}\right\}$ and $\left\{\delta_{n}\right\}$ in Theorem 3 so that

$$
\phi\left(s_{n}\right) \delta_{n} \geq 1
$$


and so (4.4) holds.

We can take $\left\{\lambda_{n}\right\}$ as in (2.8) and

$$
\delta_{n}=\frac{\left(1-r_{n}\right)^{2}}{\lambda_{n}}
$$

Then, for $n$ large,

$$
\phi\left(s_{n}\right) \delta_{n} \sim \frac{\phi\left(s_{n}\right)\left(1-r_{n}\right)^{2}}{\left(\frac{1}{1-r_{n}}\right)^{\lambda_{n-1}^{2}}}>\frac{\phi\left(s_{n}\right)}{\left(\frac{1}{1-r_{n}}\right)^{2 \lambda_{n-1}^{2}}} .
$$

Let us assume that $r_{1}, \ldots, r_{n-1}, \lambda_{1}, \ldots, \lambda_{n-1}$ and therefore $\delta_{1}, \ldots, \delta_{n-1}$ have already been defined. Then we define $r_{n}$ such that (2.7) is satisfied and such that

$$
\frac{\phi(r)}{\left(\frac{1}{1-r}\right)^{2 \lambda_{n-1}^{2}}} \geq 1, \quad r \geq r_{n}
$$

which is possible by $(2.1)$.

Once $r_{n}$ has been defined, we obtain $\lambda_{n}, s_{n}$ and $\delta_{n}$ by (2.8), (2.11) and (4.6). So we have a well-defined function $F(z)$, analytic in the unit disc, satisfying (2.2) and (2.3) in the set $E=\bigcup_{n}\left[r_{n}, r_{n}+\delta_{n}\right]$. Since (4.5) follows from (4.7) and (4.8) and (4.5) implies (2.4), the proof of Theorem 1 is complete.

\section{A preliminary result to Theorem 2}

The following result will be used in the proof of Theorem 2 .

Theorem 4. We define the sequence $\left\{r_{n}\right\}$ by

$$
\frac{1}{1-r_{n}}=\phi(n)=\Psi(n)^{1 / 3}
$$

where $\Psi(1)=1, \Psi(n)=\exp (\Psi(n-1))$ as in (1.6). Then the function defined by (2.10) satisfies

$$
S(r, F) \geq 2 T(r, F)
$$

for $r$ in a sequence of intervals $\left(t_{n}, t_{n}+\beta_{n}\right)$ such that

$$
\beta_{n} \geq \frac{1}{\Psi(n)} \text { for large } n \text {. }
$$


Proof: The sequence $r_{n}$ given by (5.1) satisfies (2.7) for a certain $\alpha$; hence $F(z)$ is a well-defined function.

In the proof of Theorem 1 we have shown that $S(r, F) \geq 2 T(r, F)$ for $r$ sufficiently close to 1 in a sequence of intervals $\left[s_{n}, s_{n}+\delta_{n}\right]$, where $s_{n}$ and $\delta_{n}$ are given by (2.11) and (2.12). We choose in particular

$$
\delta_{n}=\frac{\left(1-r_{n}\right)^{2}}{\lambda_{n}} .
$$

It remains to verify $(5.2)$.

We shall show by induction that there is $N \in \mathbf{N}$ such that

$$
\frac{\lambda_{n}}{\left(1-r_{n}\right)^{2}} \leq \phi(n+N) \leq \Psi(n+N)
$$

for every $n \in \mathbf{N}$. We assume $n$ so large that $\phi(n)$ is much bigger than $n$ and $\log \phi(n)$; for such an $n$ we find $N$ so large that (5.3) holds. Then we prove that this inequality is true for all the following terms.

In fact by (2.8) we have

$$
\begin{aligned}
\frac{\lambda_{n+1}}{\left(1-r_{n+1}\right)^{2}} & =\left\{\left[\left(\frac{1}{1-r_{n+1}}\right)^{\lambda_{n}^{2}}\right]+1\right\} \frac{1}{\left(1-r_{n+1}\right)^{2}} \\
& \leq\left(\frac{1}{1-r_{n+1}}\right)^{2 \lambda_{n}^{2}}=\exp \left\{2 \lambda_{n}^{2} \log \left(\frac{1}{1-r_{n+1}}\right)\right\} \\
& \leq \exp \left\{\frac{1}{3} \phi(n+N)^{3}\right\}=\phi(n+N+1) \\
& \leq \Psi(n+N+1),
\end{aligned}
$$

since by our hypothesis on $n$

$$
\begin{aligned}
2 \lambda_{n}^{2} \log \left(\frac{1}{1-r_{n+1}}\right) & =2 \lambda_{n}^{2} \log \phi(n+1) \\
& \leq \frac{1}{3} \phi(n+N)^{3} .
\end{aligned}
$$

This proves the inductive step, so that (5.3) holds for all large $n$. By increasing $N$ if necessary, we ensure that (5.3) is true for all $n$. Thus we obtain

$$
\delta_{n}=\frac{\left(1-r_{n}\right)^{2}}{\lambda_{n}} \geq \frac{1}{\Psi(n+N)}
$$

for some positive integer $N$ and all $n$. We conclude that (5.2) holds, writing

$$
t_{n}=s_{n-N}, \quad \beta_{n}=\delta_{n-N}, \quad n>N
$$




\section{Proof of Theorem 2}

For the function $F$ of Theorem 4 there is a sequence of intervals $\left(t_{n}, t_{n}+\beta_{n}\right)$ such that for large $n$ we have

$$
\beta_{n} \geq \frac{1}{\Psi(n)}
$$

and $S(r, F) \geq 2 T(r, F)$ for $r>r_{0}$ in $\bigcup_{n}\left(t_{n}, t_{n}+\beta_{n}\right)$. If Theorem 2 were false, we could find a sequence of disjoint intervals $\left[r_{n}, r_{n}+\delta_{n}\right]$ satisfying

$$
\delta_{n}<\frac{1-r_{n}}{e L(n)^{2}}
$$

and such that $S(r, F)=o(T(r, F))$ outside the union $\bigcup_{n}\left[r_{n}, r_{n}+\delta_{n}\right]$. Then we should have for a certain $N_{1} \in \mathbf{N}$

$$
\bigcup_{n>N_{1}}\left(t_{n}, t_{n}+\beta_{n}\right) \subset \bigcup_{k}\left[r_{k}, r_{k}+\delta_{k}\right]
$$

and, since we are considering sequences of disjoint intervals, we should conclude that

$$
\left(t_{n}, t_{n}+\beta_{n}\right) \subset\left[r_{k_{n}}, r_{k_{n}}+\delta_{k_{n}}\right] \quad n>N_{1}
$$

By the way we constructed the intervals $\left(t_{n}, t_{n}+\beta_{n}\right)$ we can assume

$$
1-t_{n+1}<\alpha\left(1-t_{n}\right)
$$

for a certain $0<\alpha<1$.

We note that since $t_{n} \rightarrow 1$ as $n$ tends to infinity we also have $r_{k_{n}} \rightarrow 1$, so that $k_{n}$ also tends to infinity as $n \rightarrow \infty$.

From (6.2), (6.3) and (6.4) we have

$$
\begin{aligned}
t_{n+1}-t_{n} & >(1-\alpha)\left(1-t_{n}\right)>(1-\alpha)\left(1-r_{k_{n}}-\delta_{k_{n}}\right) \\
& >(1-\alpha)\left(e L\left(k_{n}\right)^{2}-1\right) \delta_{k_{n}} .
\end{aligned}
$$

Since $k_{n} \rightarrow \infty$ as $n \rightarrow \infty$ and, from (2.5), $L\left(k_{n}\right)$ also tends to infinity, we can find $N_{2} \in \mathbf{N}$ such that

$$
(1-\alpha)\left(e L\left(k_{n}\right)^{2}-1\right)>1, \quad n>N_{2},
$$


so that

$$
t_{n+1}-t_{n}>\delta_{k_{n}}, \quad n>N=\max \left(N_{1}, N_{2}\right) .
$$

Therefore, for $n>N$, each interval $\left[r_{k_{n}}, r_{k_{n}}+\delta_{k_{n}}\right]$ cannot meet more than one of the intervals $\left(t_{n}, t_{n}+\beta_{n}\right)$. Hence the sequence $k_{n}$ is strictly increasing for $n>N$.

Now using (6.1), (6.2) and (6.3) we must have that, for $n$ large,

$$
\frac{1}{\Psi(n)} \leq \beta_{n} \leq \delta_{k_{n}} \leq \frac{1-r_{k_{n}}}{e L\left(k_{n}\right)^{2}} \leq \frac{1}{L\left(k_{n}\right)} \leq \frac{1}{L(n-N)}
$$

since $k_{n} \geq n-N$. Hence there is $n_{0} \in \mathbf{N}$ such that

$$
\Psi(n) \geq L(n-N), \quad n>\max \left(n_{0}, N\right),
$$

that is

$$
\Psi(n+N) \geq L(n), \quad n>n_{0} .
$$

Then we should have

$$
\Psi\left(n+N+n_{0}\right) \geq L\left(n+n_{0}\right) \geq L(n), \quad n \in \mathbf{N},
$$

which contradicts (2.5). This completes the proof of Theorem 2.

\section{References}

1. A. Fernández ARias, Some results about the size of the exceptional set in Nevanlinna's second fundamental theorem, Collect. Math. 37 (1986), 229-238.

2. A. Fernández Arias, On the size of the exceptional set in Nevanlinna theory, J. London Math. Soc. 34(2) (1986), 449-456.

3. A. Fernández Arias and F. Rodríguez Mateos, On the size of the exceptional set in Nevanlinna's second fundamental theorem for meromorphic functions in the unit disc, Rev. R. Acad. Ci. Madrid (to appear).

4. W. K. Hayman, "Meromorphic functions," Clarendon Press, Oxford, 1964

5. W. K. HAYMAn, Die Nevanlinna-Characteristic von meromorphen Funktionen und ihrer Integralen, in "Festband zum 70: Geburtstag von Rolf Nevanlinna," Springer, Berlin, 1966, pp. 16-20.

6. R. Nevanlinna, "Le théorème de Picard-Borel et la théorie des fonctions méromorphes," Gauthier-Villards, Paris, 1920. 
7. R. Nevanlinna, "Analytic functions," Springer-Verlag, Berlin, 1970.

Keywords. Characteristic function, distribution, growth, exceptional set. 1991 Mathematics subject classifications: 30D35.

Departamento de Matemáticas Fundamentales Facultad de Ciencias

Universidad Nacional de Educación a Distancia

Senda del Rey s/n

28040 Madrid

SPAIN

e-mail: arturo.fernandez@human.uned.es

Primera versió rebuda el 30 de Maig de 1995, darrera versió rebuda el 30 d'Octubre de 1995 